\title{
Epidemiology of Melioidosis in South Asia
}

\author{
C Mukhopadhyay
}

Melioidosis was first reported from Rangoon, Burma, a South East Asian country, by Whitmore and Krishnaswamyin the year 1912, from a 40 year old patient who died from an 'undescribed infective disease, somewhat resembling but really easily distinguishable from glanders'. Sixteen year later, it was diagnosed in a European tea broker from Sri Lanka, the country which is holding the $2{ }^{\text {nd }}$ South Asian Melioidosis Congress this year. This disease remained unrecognized for another 40 years, till the 1960s, before its endemicity was established in South East Asian Countries like Malaysia, Singapore and Thailand. It took another 20 more years to get the first case of melioidosis diagnosed from India, the largest South Asian country which has recently been predicted to be the 'hot spot' of the disease. A journey of almost 100 years which started from gharry ponies has been completed to genome sequencing of the causative agent of 'Tapanuli fever' or 'the black corruption of Formosa' in the year 2004.

Thailand did not feature in the early description of the disease; however, interest and awareness was stimulated in late 1980s and early 1990s with inspired collaboration and a productive program when East met West. Unfortunately, this effort remained restricted to Thailand and a few other South East Asian countries like Laos PDR and Vietnam but failed to encourage researchers from countries like India, Bangladesh, and Sri Lanka. In recent years, however, there is increasing recognition of melioidosis in the Indian subcontinent, where networking of researchers gave rise to significant enthusiasm to diagnose more cases and inspired in-depth research. Nonetheless, it is not enough - more collective and collaborative effort and funding opportunities for South Asian researchers are needed to explore the 'unknown territories' of this neglected, tropical and extremely fatal disease which should culminate into development of preventive strategies for better public health.

${ }^{1}$ Department of Microbiology, Kasturba Medical College, Manipal, India.

Address for correspondence: Dr ChiranjayMukhopadhyay, Department of Microbiology, Kasturba Medical College, Manipal, India. +919845513057 Email: chiranjay@yahoo.co.in (iDttps://orcid.org/0000-0003-0402-1143 